\title{
The Influence of Stable Boundary Layer Flows on Wind Turbine Fatigue Loads
}

\author{
Chungwook $\operatorname{Sim}^{1}$ \\ Dept. of Civil, Architectural, and Environmental Engineering, University of Texas, Austin, TX 78712, USA \\ Sukanta Basu ${ }^{2}$ \\ Dept. of Geosciences, Texas Tech University, Lubbock, TX 79409, USA \\ and \\ Lance Manuel ${ }^{3}$ \\ Dept. of Civil, Architectural, and Environmental Engineering, University of Texas, Austin, TX 78712, USA
}

\begin{abstract}
Near-neutral atmospheric stability conditions form the basis for wind turbine design. This is surprising since such near-neutral conditions occur in so-called transition periods only twice each day (around sunrise and sunset). Unstable conditions occur during the day and stable conditions occur generally at night. During nighttime stable conditions, turbulence is typically generated by shear and destroyed by negative buoyancy. Wind shear (both magnitude and direction) under stable conditions is much larger in comparison to that during neutral conditions. Moreover, stable boundary layer (SBL) flows are often accompanied by low-level jets (LLJs); these LLJs can be low enough to impact today's large utility-scale turbines and thus influence loads. This study compares turbulence, turbine loads, and accumulated fatigue damage for a utility-scale wind turbine in stable versus neutral atmospheric conditions. Our focus is on the varying simulated atmospheric flows that result from (i) different surface cooling rates (which control buoyancy destruction); and (ii) different geostrophic winds (which control shear generation). Inflow turbulence time series are generated and applied over the rotor plane of a 90-meter hub-height 5MW wind turbine based on Large-Eddy Simulation (LES) with refined dynamic sub-grid scale modeling; similarly, neutral boundary layer flows are generated using conventional Fourier techniques for comparison. These simulated wind velocity fields are then fed into an aeroelastic model of the selected wind turbine and turbine fatigue loads are analyzed. Some differences are seen between fatigue loads resulting from neutral versus stable conditions but these are not significant especially when missing high-frequency content in the inflow turbulence from LES-generated flows is augmented by fractal interpolation.
\end{abstract}

\section{Introduction}

$\mathrm{O}$ ver the past two decades, there has been considerable wind energy development in the Great Plains regions of the U.S. where nocturnal low-level jets (LLJs) occur quite frequently. The peaks (local wind maxima) of LLJs are typically centered around 100-1,000 m above the ground level and make the Great Plains' wind resources favorable for wind energy production. At the same time, the presence of LLJs can significantly modify vertical shear and night-time turbulence environments in the vicinity of wind turbine hub heights can have a detrimental effects on rotors. ${ }^{1}$ Thus, accurate numerical modeling and forecasting of LLJs is needed for robust wind turbine design and for more reliable power generation prediction. Since stable stratification is a prerequisite for the occurrence of nocturnal LLJs, this then requires an improved modeling capability of stable boundary layers (SBLs). An example of such modeling using Large-Eddy Simulation (LES) is demonstrated in Fig. 1; the dark red bands of

${ }^{1}$ Graduate Research Assistant

${ }^{2}$ Assistant Professor

${ }^{3}$ Associate Professor 
higher wind speeds in the 100-250 m range represent a low-level jet. Sporadic bursts of turbulence in the midst of otherwise quiescent stable stratified conditions are also clearly visible in Fig. 1(b). Hub heights and rotor tips of contemporary wind turbines are also depicted in the figures; a $5 \mathrm{MW}$ turbine rotor will experience strong shear over a 60-120 m vertical band (in Fig. 1(a), the lower rotor tip of a 5MW turbine experiences a wind speed of around 8 $\mathrm{m} / \mathrm{s}$, whereas the upper tip experiences a wind speed close to $11 \mathrm{~m} / \mathrm{s}$ ).

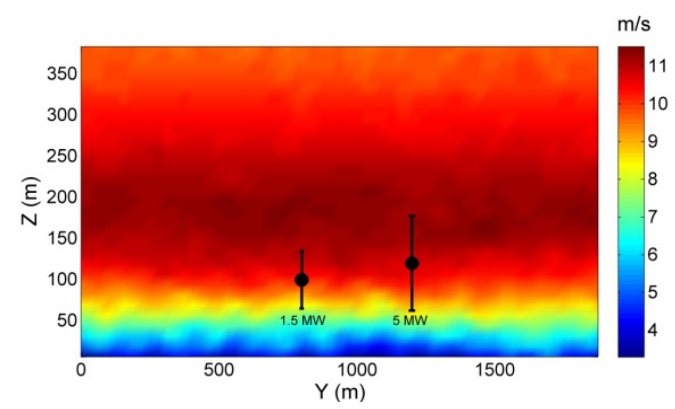

(a)

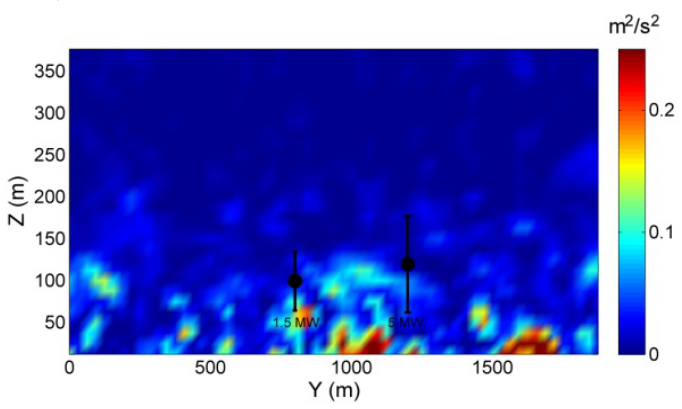

(b)

Figure 1. (a) Lateral cross-section of mean wind speed field generated by an LES model; and (b) LES-resolved turbulence kinetic energy field.

In the International Electrotechnical Commission (IEC) guidelines for wind turbine design ${ }^{2}$, the recommended "normal turbulence model" assumes stationary spectral representations can serve as the basis for stochastic simulation of inflow turbulence field. Only near-neutral stability conditions are considered in such simulations. Recent studies suggest that significant fatigue damage can occur from coherent structures that are produced in flows common in the stable, nocturnal atmospheric boundary layer. ${ }^{3}$ Moreover, the non-stationary character of wind velocity processes, not represented in the IEC turbulence models, can be another important factor in predicting wind turbine loads. From the present study, LES-generated data sets will, for the first time, be employed in turbine load simulations. ${ }^{4}$ We shall demonstrate that consideration of non-neutral atmospheric conditions (especially stable flows) in establishing turbine fatigue loads is possible - only a very limited amount of published research is available in this regard. For comparison purposes, neutral boundary layer velocity fields will also be simulated using conventional Fourier techniques as is possible using TurbSim. ${ }^{5}$

Fatigue loads on a utility-scale ( $5 \mathrm{MW}$ ) turbine model for a few representative simulated wind inflow turbulence fields are of interest in this study. Stochastic simulations of the aeroelastic response of the selected turbine will be carried out using the software, $\mathrm{FAST}^{6}$; these simulations are used to study time series of turbine loads - blade bending loads and tower loads are considered - that are then used to statistically estimate fatigue load spectra.

\section{Inflow and Wind Turbine Simulation}

\section{A. LES with refined dynamic sub-grid scale modeling}

Large-eddy simulation (LES) is at present the most efficient technique available for high Reynolds number flow simulations, such as for atmospheric boundary layer (ABL) simulations, in which the larger scales of motion are resolved explicitly and the smaller ones are modeled. Over the past three decades, the field of LES for the ABL has evolved quite dramatically. LES has enabled researchers to probe various boundary layer flows by generating unprecedented high-resolution four-dimensional turbulence data. As a consequence, we have gained a better understanding of some fairly complex ABL phenomena.

In rotation-influenced ABLs, the equations governing the conservation of momentum (using the Boussinesq approximation) and temperature are:

$$
\frac{\partial \tilde{u}_{i}}{\partial t}+\frac{\partial\left(\tilde{u}_{i} \tilde{u}_{j}\right)}{\partial x_{j}}=-\frac{\partial \tilde{p}}{\partial x_{i}}-\frac{\partial \tau_{i j}}{\partial x_{i}}+\delta_{i 3} g \frac{(\tilde{\theta}-\langle\tilde{\theta}\rangle)}{\theta_{0}}+f_{c} \varepsilon_{i j 3} \tilde{u}_{j}+F_{i}
$$




$$
\frac{\partial \tilde{\theta}}{\partial t}+\frac{\partial\left(\tilde{u}_{j} \tilde{\theta}\right)}{\partial x_{j}}=-\frac{\partial q_{j}}{\partial x_{j}}
$$

where $t$ refers to time; $x_{j}$ is the spatial coordinate in the direction, $j ; u_{j}$ is the velocity component in the direction, $j ; \theta$ is potential temperature; $\theta_{0}$ is the reference surface potential temperature; $p$ is dynamic pressure; $\delta_{i 3}$ is the Kronecker delta; $\varepsilon_{i j 3}$ is the alternating unit tensor; $g$ is the gravitational acceleration; $f_{c}$ is the Coriolis parameter; and $F_{i}$ is a forcing term (e.g., geostrophic wind).

Molecular dissipation and diffusion have been neglected since the Reynolds number of the ABL is very high and no near-ground viscous processes are resolved. Note that $\langle$.$\rangle is used to define a horizontal plane average; also$ the tilde (i.e., “ $")$ above some variables in Eqs. (1a) and (1b) denotes a spatial filtering operation, using a filter of characteristic width, $\Delta_{f}$. These filtered equations are now amenable for numerical solution on a grid of mesh size, $\Delta_{g}$, considerably larger than the smallest scale of turbulent motion (the so-called Kolmogorov scale). The effects of the unresolved scales (smaller than $\Delta_{f}$ ) on the evolution of $\tilde{u}_{i}$ and $\tilde{\theta}$ appear in the subgrid-scale (SGS) stress, $\tau_{i j}$ (in Eq. (1a)) and the SGS flux $q_{j}$ (in Eq. (1b)), respectively; these are defined as follows: $\tau_{i j}=\tilde{u_{i}} \tilde{u}_{j}-\tilde{\tilde{u}_{i}} \tilde{u}_{j}$, and $q_{j}=\widetilde{u_{i}} \dot{\theta}-\mathscr{U}_{i} \tilde{\theta}$. Note that the SGS stress and flux quantities are unknown and must be parameterized (using a SGS model) as a function of the resolved velocity and temperature fields.

Eddy viscosity models, the most popular SGS models, use the "gradient hypothesis" and formulate the SGS stress tensor (the deviatoric part) as follows ${ }^{7,8}$ :

$$
\tau_{i j}-\frac{1}{3} \tau_{k k} \delta_{i j}=-2 v_{t} \tilde{S}_{i j}
$$

where $S_{i j}$ is the resolved strain rate tensor and $v_{t}$ denotes the eddy viscosity. From a dimensional analysis, $v_{t}$ can be interpreted as the product of a characteristic velocity scale and a characteristic length scale ${ }^{8}$. Different eddyviscosity formulations basically use different velocity and length scales. The most popular eddy viscosity formulation is the Smagorinsky model ${ }^{7}$ :

$$
v_{t}=\left(C_{S} \Delta_{f}\right)^{2}|\tilde{S}|
$$

where $C_{S}$ is the so-called Smagorinsky coefficient, which is adjusted empirically or dynamically to account for shear, stratification, and grid resolution, and $\left|S_{i j}\right|$ is the magnitude of the resolved strain rate tensor. Similar to the SGS stresses, eddy-diffusivity models are used for the SGS heat fluxes as follows:

$$
q_{i}=-v_{h t} \frac{\partial \tilde{\theta}}{\partial x_{i}}=-\frac{v_{t}}{\operatorname{Pr}_{S G S}} \frac{\partial \tilde{\theta}}{\partial x_{i}}
$$

where $\operatorname{Pr}_{S G S}$ the SGS Prandtl number.

The values of the Smagorinsky-type SGS model parameters, $C_{S}$ and $\operatorname{Pr}_{S G S}$, are well established for homogeneous isotropic turbulence. ${ }^{9}$ However, the value of $C_{S}$ is expected to decrease with increasing mean shear and stratification. This expectation has been confirmed by various recent field studies. In order to account for shear and stratification, application of the traditional eddy-viscosity model in LES of ABL flows (with strong shear near the ground and temperature-driven stratification) has traditionally involved the use of various types of wall-damping functions and stability corrections, which are either based on the phenomenological theory of turbulence or empirically derived from observational data. Similar a priori prescriptions exist also in the case of eddy-diffusivity SGS models. 
An alternative approach is to use the "dynamic" SGS modeling approach. In this approach, one computes the value of the unknown SGS coefficients (e.g., $C_{S}$ in the Smagorinsky-type eddy-viscosity models) dynamically at every time and every position in the flow. By looking at the dynamics of the flow at two different resolved scales and assuming scale similarity as well as scale invariance of the model coefficient, one can optimize its value. ${ }^{9,10}$ Thus, the dynamic model avoids the need for a priori specification and tuning of the coefficient because it is evaluated directly from the resolved scales in an LES.

Recently, Basu and Porté-Agel ${ }^{4}$ proposed a refined dynamic modeling approach (called the "locally-averaged scale-dependent dynamic" or LASDD SGS modeling approach) for ABL simulations. The potential of the LASDD SGS model was made clear in large-eddy simulations of the neutral boundary layer ${ }^{11}$, of the stable boundary layer ${ }^{4}$, and of a complete diurnal cycle ${ }^{12}$.

In the present study, we utilize the LASDD model to generate stably stratified inflow conditions for wind turbine load calculations using an aeroelastic model.

\section{B. Inflow Simulation}

In atmospheric large-eddy simulations, idealized or observed soundings (i.e., 1-D vertical profiles) of wind speed and other environmental variables (such as temperature, moisture, etc.) in conjunction with small-scale 3-D perturbations (random noise) are typically used to generate initialization fields. With the help of the Navier-Stokes equations (Eq. (1)), these fields are then evolved in time under the constraints of certain large-scale forcing terms (e.g., geostrophic wind) and boundary conditions (e.g., prescribed land-surface temperature is often used as the lower boundary condition). Usually, it takes about an hour of simulation (depending on the characteristics of the boundary layer to be simulated) to generate realistic turbulence (e.g., for reasonable representation of the inertial range of spectra). However, it can take a few hours of simulation to generate quasi-steady state boundary layer conditions. For realistic stable boundary layer simulations, one needs to run an LES code for O(12) hours to reach quasi-steady state conditions (we note that the boundary layer meteorology community is aware that certain turbulence statistics under stably stratification never reach a steady-state condition - a cogent discussion on this topic is beyond the scope of this paper).

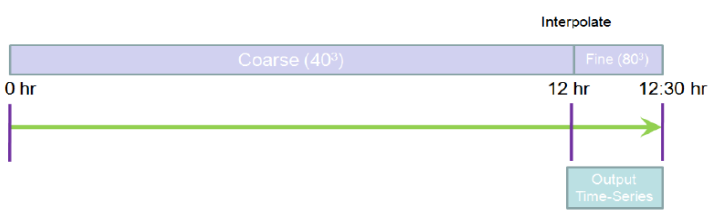

(a)

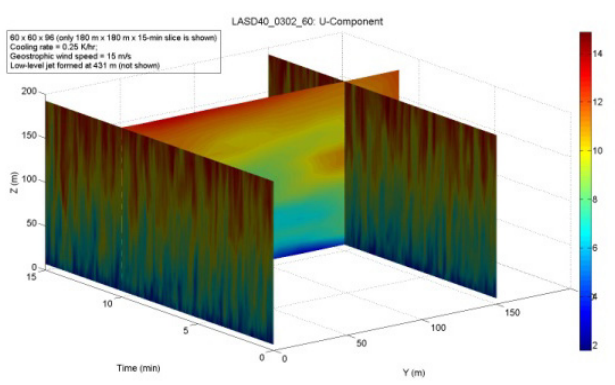

(b)

Figure 2. (a) Two phases of the LES flow generation; and (b) a slice of the last 15 minutes of phase II longitudinal velocity wind field generated from LES.

High-resolution LES runs are computationally very expensive, especially for durations of $O(12)$ physical hours. For this reason, in this work we carry out the simulations in two phases (see Fig. 2(a)). In phase I, coarse runs (with a grid resolution of $20 \mathrm{~m}$ ) of 12 -hour durations are performed using a time step of 0.2 seconds. Then, in phase II, the final 3-D fields from the phase I simulations are used as initial fields and new simulations are run for 30 minutes (with a time step of 0.1 seconds). In order to create higher resolution (finer than $20 \mathrm{~m}$ ) LES data, we first apply a cubic spline interpolation to the final 3-D fields of the phase I simulations to produce $13.3 \mathrm{~m}$ resolution initial fields. Full-field wind files for 3-D velocity components are output from the last 15 minutes of these 30-minute phase II simulations at a frequency of $2.5 \mathrm{~Hz}$ (i.e., every 0.4 seconds). For both the phases of our simulations, we kept a fixed domain size of $800 \mathrm{~m} \times 800 \mathrm{~m} \times 1,260 \mathrm{~m}$. Figure 2(b) shows a $180 \mathrm{~m} \times 180 \mathrm{~m}(y-z$ plane $)$ slice of the 
longitudinal velocity $(U)$ taken at one time instant from the last 15-minute time history segment of the simulated wind field (with a grid resolution of $13.3 \mathrm{~m}$ ); also shown are the 15 -min time series for $U$ versus vertical elevation (z) for points laterally separated by $150 \mathrm{~m}$.

In this study, we systematically varied geostrophic winds (a large-scale forcing term related to mesoscale pressure gradient force) and surface temperature cooling rates (a lower boundary condition that strongly influences the level of stratification in stable boundary layers). Table 1 shows case ID designations for all the different combinations (geostrophic wind speed: GWS, cooling rate: CR) simulated as part of this study. The first two digits in the ID indicate the geostrophic wind ( 01 indicates $\mathrm{GWS}=5 \mathrm{~m} / \mathrm{s}$, etc.) while the next two digits indicate the cooling rate $(01$ indicates $\mathrm{CR}=0.01 \mathrm{~K} / \mathrm{hr}$, etc.). The last two digits relate to the grid spacing (e.g., 40 indicates a 20 $\mathrm{m}$ grid spacing).

Table 1: Various simulation cases and associated bulk characteristics of the simulated boundary layers

\begin{tabular}{|c|c|c|c|c|c|c|c|}
\hline Case ID & $\begin{array}{c}\text { Grid-Resolution } \\
\text { (m) }\end{array}$ & $\begin{array}{l}\text { GWS } \\
(\mathrm{m} / \mathrm{s})\end{array}$ & $\begin{array}{c}\text { CR } \\
(\mathbf{K} / \mathbf{h r})\end{array}$ & $\begin{array}{c}\text { LLJ H } \\
\text { (m) }\end{array}$ & $\begin{array}{c}\text { LLJ WS } \\
(\mathrm{m} / \mathrm{s})\end{array}$ & $\begin{array}{c}\text { Power Law } \\
\text { Exponent }\end{array}$ & $\begin{array}{c}\text { Directional } \\
\text { Change (deg) }\end{array}$ \\
\hline $0101-40$ & 20.0 & 5 & 0.01 & 250 & 5.6 & 0.26 & 6.7 \\
\hline 0101-60 & 13.3 & 5 & 0.01 & 245 & 5.6 & 0.24 & 7.1 \\
\hline $0102-40$ & 20.0 & 5 & 0.25 & 150 & 5.9 & 0.34 & 28.3 \\
\hline $0102-60$ & 13.3 & 5 & 0.25 & 153 & 5.9 & 0.37 & 29.3 \\
\hline $0103-40$ & 20.0 & 5 & 0.50 & 130 & 5.9 & 0.30 & 31.2 \\
\hline $0201-40$ & 20.0 & 10 & 0.01 & 450 & 10.9 & 0.21 & 4.0 \\
\hline $0201-60$ & 13.3 & 10 & 0.01 & 458 & 10.9 & 0.21 & 3.8 \\
\hline $0202-40$ & 20.0 & 10 & 0.25 & 270 & 11.8 & 0.35 & 11.4 \\
\hline $0202-60$ & 13.3 & 10 & 0.25 & 272 & 11.8 & 0.35 & 10.5 \\
\hline $0203-40$ & 20.0 & 10 & 0.50 & 310 & 12.5 & 0.40 & 17.7 \\
\hline $0301-40$ & 20.0 & 15 & 0.01 & 610 & 16.8 & 0.18 & 3.9 \\
\hline $0301-60$ & 13.3 & 15 & 0.01 & 630 & 16.7 & 0.19 & 3.0 \\
\hline $0302-40$ & 20.0 & 15 & 0.25 & 430 & 17.8 & 0.32 & 7.7 \\
\hline $0302-60$ & 13.3 & 15 & 0.25 & 431 & 17.8 & 0.28 & 4.9 \\
\hline $0303-40$ & 20.0 & 15 & 0.50 & 350 & 17.8 & 0.37 & 10.1 \\
\hline
\end{tabular}

In order to provide neutral boundary layer flows whose effects on turbine loads could be directly contrasted with the stable boundary layer flows generated using LES, the Fourier-based stochastic turbulence simulation code, TurbSim, was used together with target turbulence power spectra (Kaimal type) and coherence functions. The rotor plane of the selected 5MW turbine, with a rotor diameter of $126 \mathrm{~m}$, was represented as $9 \times 9$ grid that covers a square area of side $160 \mathrm{~m}$, centered at the rotor hub. A time step of 0.4 seconds was used in the neutral BL flow simulations to match the time step from LES. Note that the wind speed at hub height $(90 \mathrm{~m})$ for the LES case with a geostrophic wind speed of $15 \mathrm{~m} / \mathrm{s}$ and a cooling rate of $0.25 \mathrm{~K} / \mathrm{hr}$ was used as the target hub-height wind speed for neutral boundary inflow produced by TurbSim.

\section{Wind Turbine Model}

A 5MW wind turbine model developed at the National Renewable Energy Laboratory (NREL) that closely represents utility-scale wind turbines being manufactured today is used for the aeroelastic response simulations in this study ${ }^{13}$. The turbine is a variable-speed, collective pitch-controlled machine and has a hub 90 meters above the ground level with a rotor diameter of 126 meters. We are interested in studying fatigue loads that result from neutral versus stable boundary layer flows. In particular, we choose to study the out-of-plane bending moment at a blade 
root and the fore-aft tower bending moment at the base. Ten-minute time series for these loads are simulated using FAST $^{6}$ and fatigue loads evaluated.

\section{Grid Resolution and Fractal Interpolation for Large Eddy Simulations of SBL}

In this study, for large-eddy simulations we used grid resolutions of $\mathrm{O}(10) \mathrm{m}$. Since spatial and (implicit) temporal filtering operations are intimately related in LES, the grid resolution basically dictated the level of highfrequency content in the generated time series. For all our runs, temporal frequencies of $\mathrm{O}(>0.2) \mathrm{Hz}$ had minimal energy, as would be anticipated. However, in routine wind turbine load calculations, for example, using TurbSim for neutral conditions, it is not uncommon to use synthetically generated velocity time series of frequency $\mathrm{O}(20) \mathrm{Hz}$. To simulate time series of $20 \mathrm{~Hz}$ frequency using a large-eddy model would require a grid-resolution of $\mathrm{O}(0.1) \mathrm{m}$. At the present time, this is a computationally daunting task. To the best of our knowledge, it is not generally believed that we even need to represent frequencies of $\mathrm{O}(20) \mathrm{Hz}$ in the inflow in order to characterize the loads appropriately. In the near future, we hope to address this question by systematically running LES at different grid resolutions (and, thus, with variable effective frequency content) and assessing differences in turbine load statistics.

Instead of performing computationally expensive high-resolution large-eddy simulations, one could also enhance the high-frequency content of coarse-resolution LES data by using the so-called fractal interpolation technique (FIT). FIT is an iterative affine mapping procedure that may be used to construct synthetic deterministic small-scale fields from a few given large-scale interpolating points. ${ }^{14}$ FIT is computationally very inexpensive and, more importantly, FIT preserves the higher-order moments and non-Gaussian probability density function of the velocity increments. ${ }^{14}$

In this study, we test both the aforementioned approaches: (1) running LES at a higher resolution (e.g., case 0302-60; resolution of $13.3 \mathrm{~m}$ ) compared to that of the base inflow (e.g., 0302-40; resolution of $20 \mathrm{~m}$ ); and (2) enhancing the frequency content of the base inflow (e.g., 0302-40; resolution of $20 \mathrm{~m}$ ) by applying the FIT technique (e.g., case 0302-40-Fractal).

Comparing power spectra of hub-height longitudinal turbulence for the neutral case (Kaimal) and the stable base inflow case (0302-40) inFig. 3, it is clear that low-frequency energy is similar but high-frequency energy is lacking in the stable case with the coarse grid resolution $(20 \mathrm{~m})$. However, simulation with a finer grid resolution $(13.3 \mathrm{~m})$ as well as the use of fractal interpolation (0302-40-Fractal) increases the energy at high frequencies. For the fractal interpolation case, especially, the energy is now comparable with the neutral case.
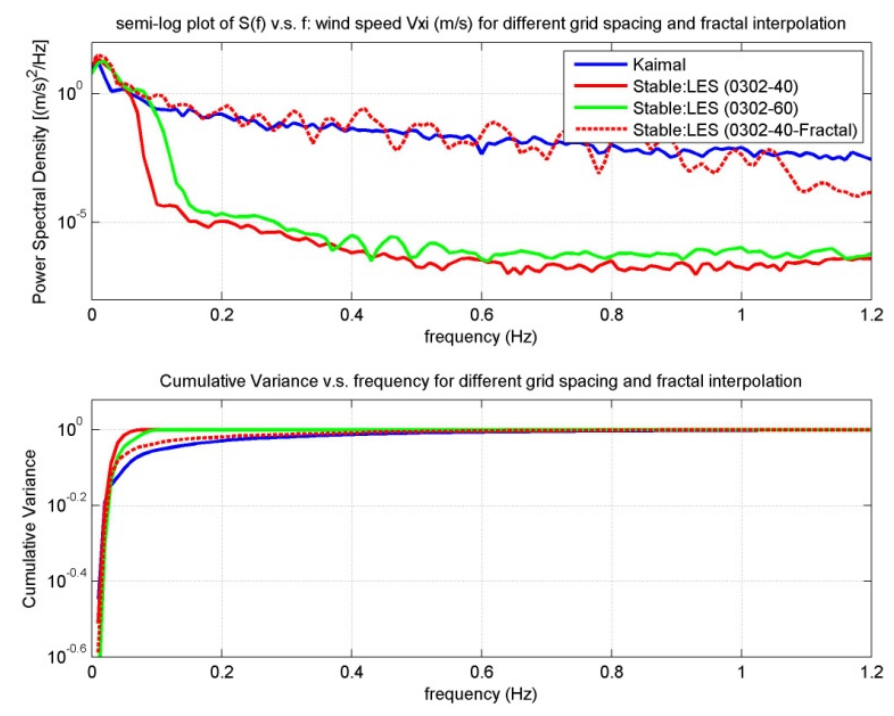

Figure 3. Power spectra and cumulative variance versus frequency plots of longitudinal velocity at hub height for various turbulence generation options. 

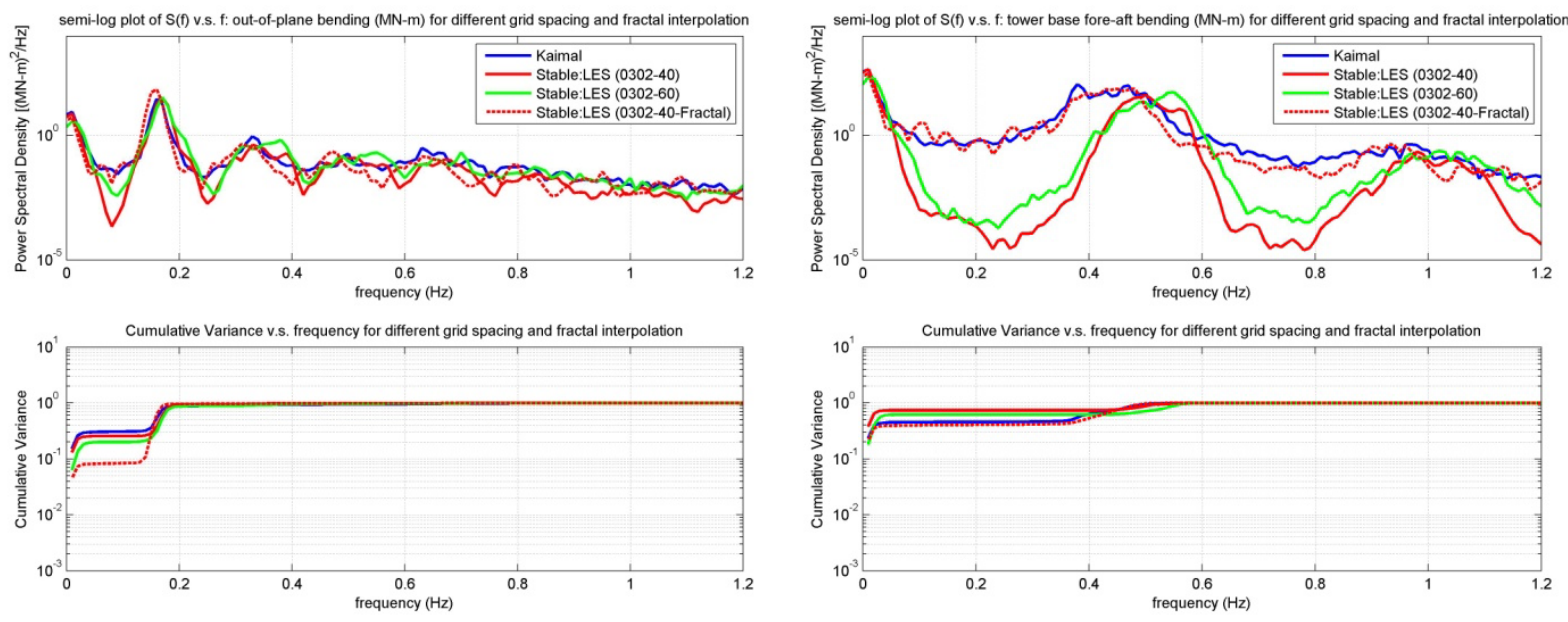

(a)

(b)

Figure 4. Power spectra and cumulative variance versus frequency for turbine loads with different turbulence modeling techniques for: (a) blade-root out-of-plane bending moment; and (b) fore-aft tower base out-of-plane bending moment.

Despite the difference in turbulence, power spectra of rotor loads in Fig. 4(a) are remarkably similar for each modeling technique. However, without the fractal interpolation, tower resonant modes seen in Fig. 4(b) are more clearly evident in the stable case since turbulence inflow background (low-frequency) energy is significantly dominant over resonant energy when high-frequency energy is absent as is the case with the LES-generated SBL flows; this also explains the contrasting power spectra relative to the neutral case. Also, with the fractal interpolation, power spectra of the tower loads are comparable for the neutral and stable cases. Clearly applying FIT to LES-generated turbulence for stable boundary layer flows leads to turbine loads that have smaller differences for neutral versus stable conditions.

\section{Fatigue Loads for Stable versus Neutral Boundary Layer Flows}

Stress or load cycle histograms can be established from time series of wind turbine loads by various means including the rainflow cycle-counting algorithm. ${ }^{15}$ A schematic diagram showing the procedures for calculating the histograms and equivalent fatigue loads for a specific Wohler exponent, $m$, and an outline of the rainflow cyclecounting algorithm are presented in Fig. 5.

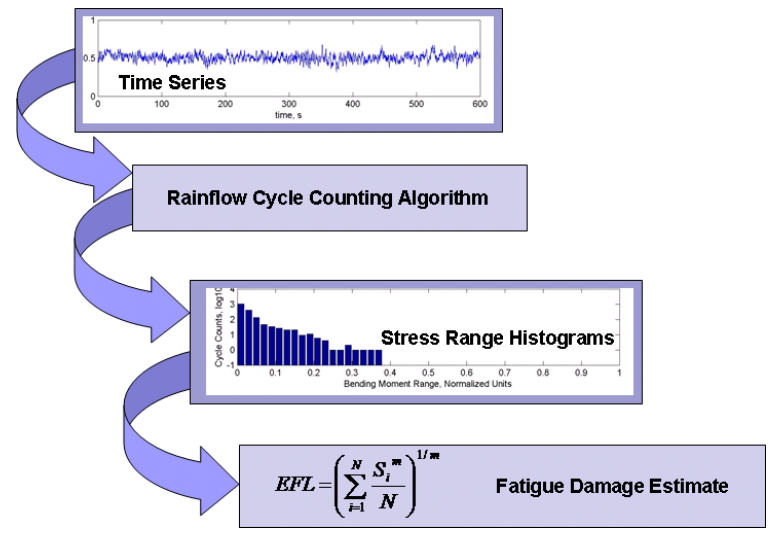

(a)

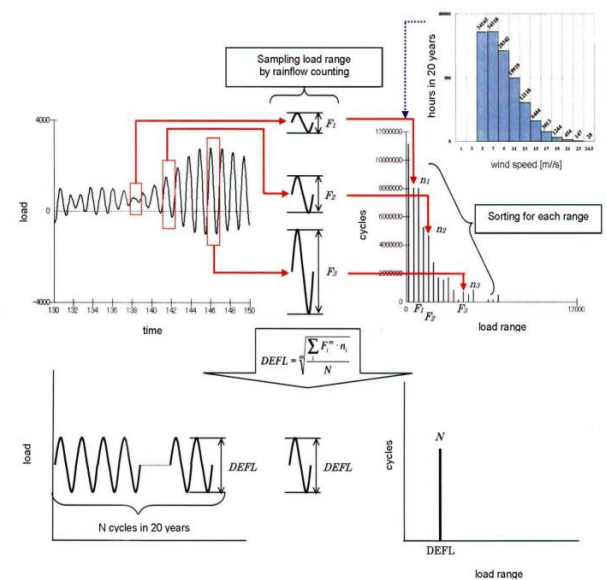

(b)

Figure 5. (a) Procedure to compute equivalent fatigue damage; (b) Rainflow Cycle-Counting Procedure. 


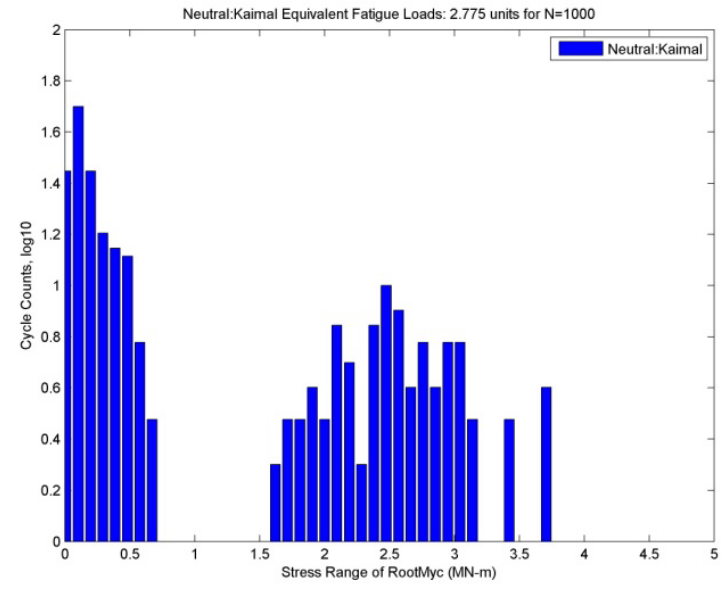

(a)

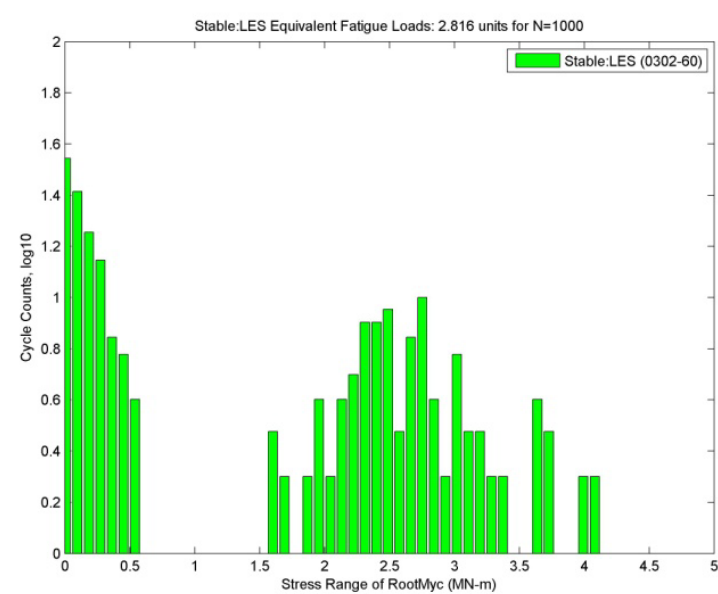

(c)

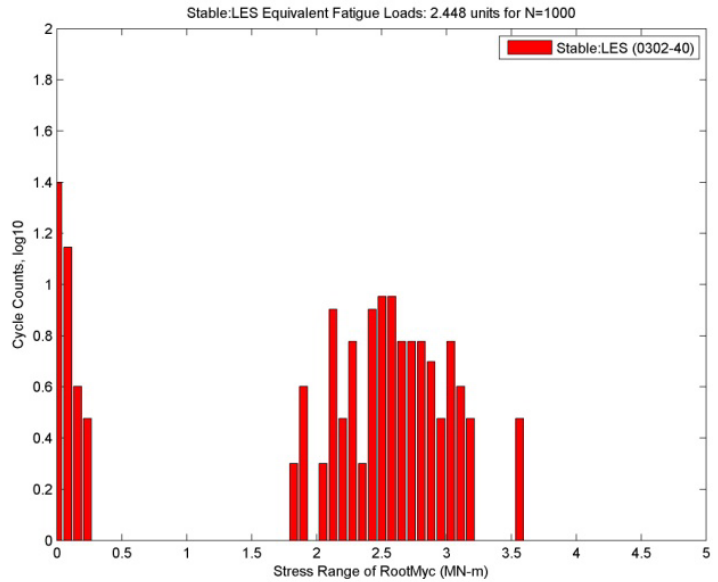

(b)

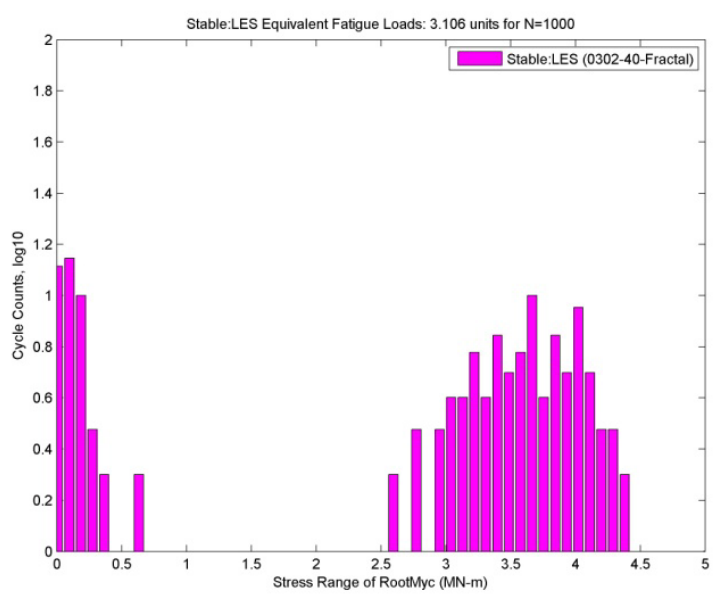

(d)

Figure 6. Fatigue damage histograms and equivalent fatigue loads for the out-of-plane bending moment at a blade root in (a) neutral inflow; (b) stable base inflow (0302-40); (c) stable inflow with finer grid spacing case (0302-60); and (d) stable inflow with fractal interpolation technique (0302-40-Fractal).

For the SBL case with a geostrophic wind speed of $15 \mathrm{~m} / \mathrm{s}$ and a cooling rate of $0.25 \mathrm{~K} / \mathrm{hr}$ and the corresponding matching neutral case (i.e., with matching mean wind speed at hub height), Fig. 6 shows histograms of the blade root out-of-plane bending moment loads for TurbSim-generated neutral boundary layer flows as well as for LES-generated stable boundary layer flows for the base case $(20 \mathrm{~m}$ resolution), for a finer grid (13.3 $\mathrm{m}$ resolution), and for the fractal interpolation case. For the different load time series, an equivalent fatigue load (with $m=10$ ) is computed assuming 1,000 cycles and is reported at the top of each plot. The equivalent fatigue loads in Figs. 6(b) and (c) are slightly smaller than in Fig. 6(a), the neutral case. However, after fractal interpolation is applied, the equivalent fatigue load leads to a larger number of large-amplitude load cycles compared to LES cases without interpolation, as seen in Fig. 6(d). In general, however, the equivalent fatigue loads appear to be quite comparable for neutral and stable cases when one considers blade loads.

Figure 7 shows similar histograms (to those shown in Fig. 6) but for the fore-aft tower bending moment at the base. The stress histograms suggest that fatigue loads for the stable case are considerably lower than those for the neutral case unless fractal interpolation is applied to the LES-generated flows. The fractal interpolation leads to an increase of almost $40 \%$ in equivalent fatigue loads $(m=3)$ in the stable case. Even after the FIT adjustment, however, equivalent fatigue loads for the stable case are still about $10 \%$ smaller than those for the neutral case. 


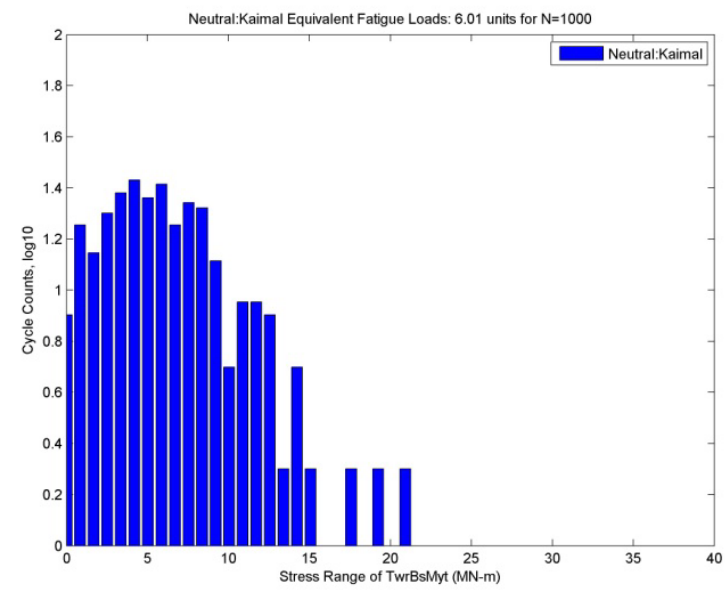

(a)

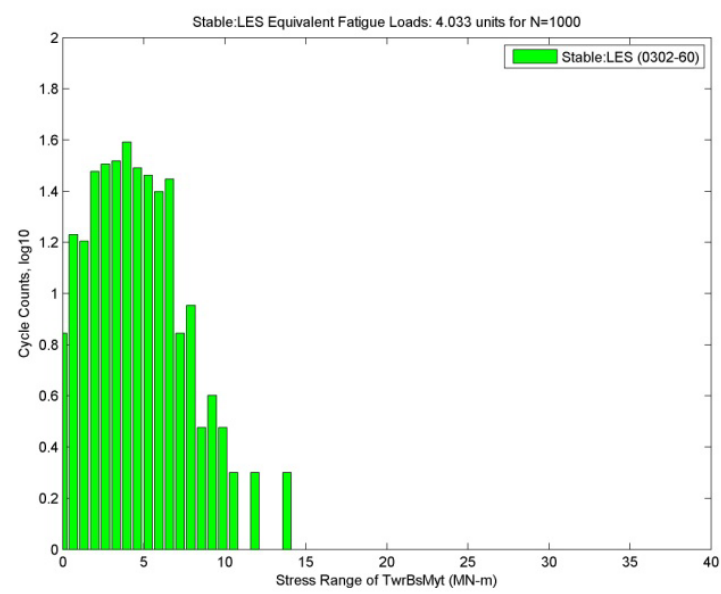

(c)

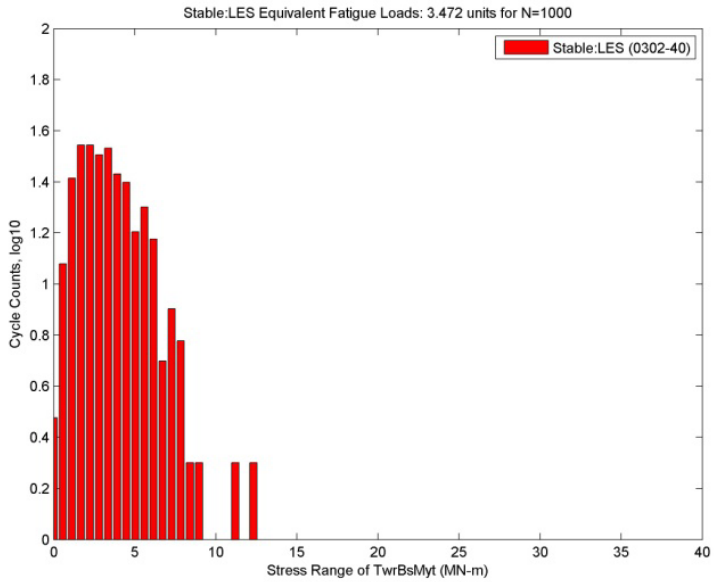

(b)

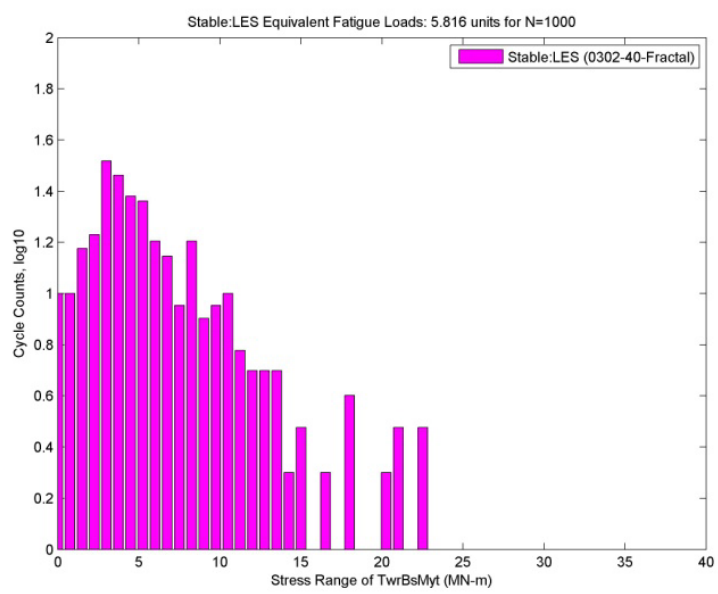

(d)

Figure 7. Fatigue damage histograms and equivalent fatigue loads for the fore-aft tower bending moment at the base in (a) neutral inflow; (b) stable base inflow (0302-40); (c) stable inflow with finer grid spacing case (0302-60); and (d) stable inflow with fractal interpolation technique (0302-40-Fractal).

\section{Sensitivity Study on Parameters of the Stable Boundary Layer Flows}

Two bulk parameters that characterize the SBL flows that were generated using LES are the geostrophic wind speed and surface temperature cooling rate. By varying the geostrophic wind speed from 5 to $15 \mathrm{~m} / \mathrm{s}$, this parameter's influence on loads could be studied. In a similar manner, by varying the surface temperature cooling rate from 0.01 to $0.50 \mathrm{~K} / \mathrm{hr}$, this parameter could also be studied.

\section{A. Geostrophic Wind Speed}

Figure 8(a) shows the power spectral density of the longitudinal velocity component at hub height for the three different geostrophic wind speeds; a neutral case is also shown for comparison. In all of the stable flow cases, energy at lower frequencies is comparable and similar to that for the neutral case but is significantly lower at high frequencies. Consistent with the larger forcing at higher geostrophic wind speeds, the variance is largest for the 15 $\mathrm{m} / \mathrm{s}$ geostrophic wind. Figure 8(b) shows power spectra for the fore-aft tower base bending moment load for three different cases. The resonant component (seen at frequencies above approximately $0.3 \mathrm{~Hz}$ ) grows with decreasing geostrophic wind speed. This is possibly because the background energy is lowest compared to the neutral case for lowest geostrophic wind speeds. 

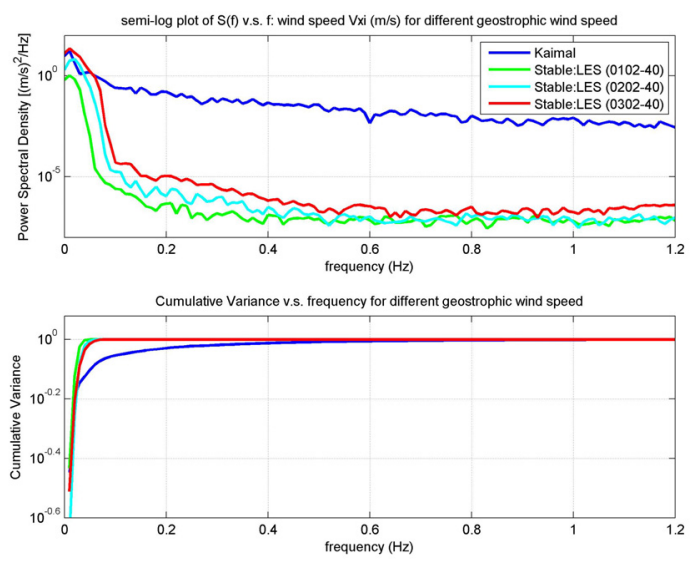

(a)
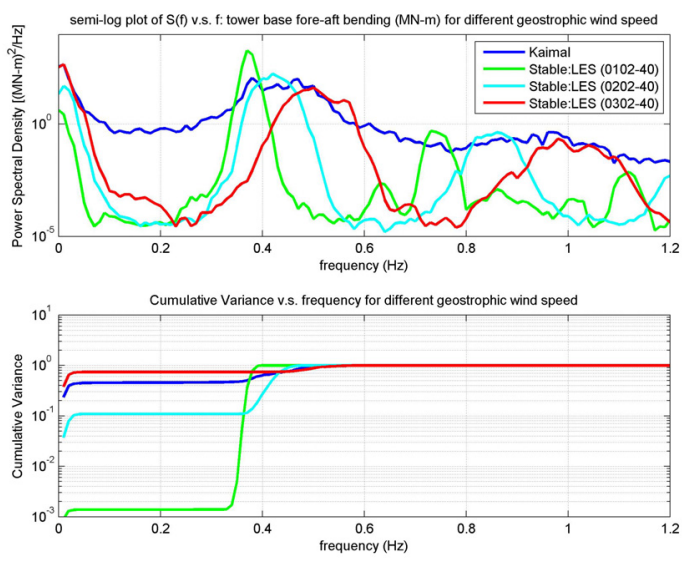

(b)

Figure 8. Variation with geostrophic wind speed for SBL flows of (a) power spectra and cumulative variance versus frequency of longitudinal velocity at hub height; and (b) power spectra and cumulative variance versus frequency of fore-aft tower base out-of-plane bending moment. Results for a neutral case are also shown for comparison.

\section{B. Surface Temperature Cooling Rate}

Figure 9 shows the power spectral density of the longitudinal velocity component at hub height and associated fore-aft tower base bending moment for the three different surface temperature cooling rates; a neutral case is also shown for comparison. Figure 9(a) suggests that with decrease in the cooling rate, energy shifts slightly to somewhat higher frequencies. Changes in cooling rates do not seem to affect tower loads greatly; perhaps a slight increase in high-frequency energy is evident with lower cooling rates in the tower loads as was the case with the inflow turbulence.
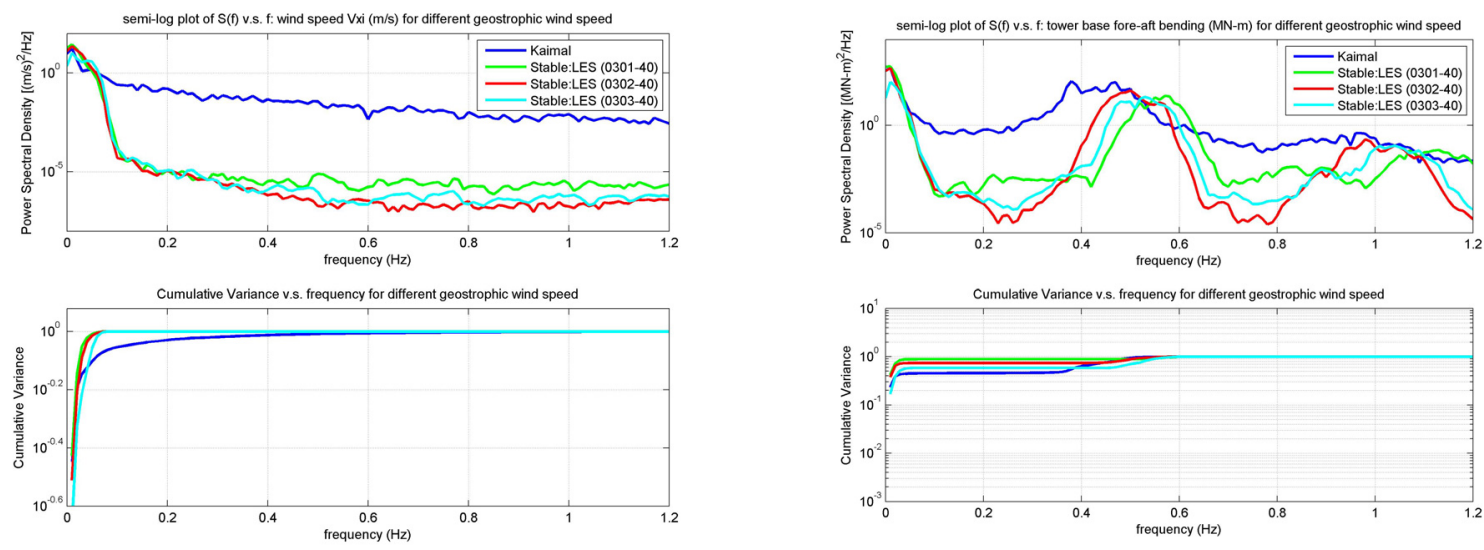

(a)

(b)

Figure 9. Variation with surface temperature cooling rate for SBL flows of (a) power spectra and cumulative variance versus frequency of longitudinal velocity at hub height; and (b) power spectra and cumulative variance versus frequency of fore-aft tower base out-of-plane bending moment. Results for a neutral case are also shown for comparison. 


\section{Conclusions}

Near-neutral atmospheric stability conditions are all that are addressed in today's wind turbine design guidelines. It has been suggested that stable boundary flows and accompanying high wind shears and low-level jets occur at those times when a disproportionately higher number of turbine faults have occurred. Large-eddy simulation (LES) offers a means of simulating stable boundary layer (SBL) flows that can be fed into turbine aeroelastic response simulations in a similar manner to how Fourier-based procedures are used to generate neutral boundary layer flows.

Due to spatial resolution limitations imposed by prohibitive computational costs, LES-generated flows are deficient in energy at high frequencies. However, fractal interpolation can help resolve high-frequency turbulence that might influence rotor and tower loads on a turbine. It was shown that such fractal interpolation introduces significant portions of high-frequency energy in inflow turbulence and turbine loads leading to comparable levels to neutral conditions. Therefore, it is reasonable to employ fractal interpolation while using LES for SBL flows in similar turbine load studies. Fatigue damage predictions for neutral and stable boundary flows also suggest comparable levels of rotor loads after fractal interpolation is introduced; for tower loads, neutral flows lead to higher equivalent fatigue loads.

By varying surface temperature cooling rates and geostrophic winds for LES, simulated turbine loads have been studied to assess the influence of different bulk characteristics of the atmospheric boundary layer on turbine loads. In general, tower loads from SBL flows with lower geostrophic winds have pronounced resonant peaks but lack high-frequency energy; variation in cooling rate has considerably less influence on tower loads.

\section{Acknowledgements}

The authors are pleased to acknowledge financial support received from Sandia National Laboratories (by way of Contract No: 743358), from the Texas Higher Education Coordinating Board's Advanced Research Program (Grant No. 003658-0100-2007), and from the National Science Foundation (Grant No: ATM-0748606).

\section{References}

${ }^{1}$ Kelley,N., Shirazi, M., Jager, D., Wilde, S., Adams, J., Buhl, M., Sullivan, P., and Patton, E., "Lamar Low-Level Jet Project Interim Report,” National Renewable Energy Laboratory, NREL/TP-500-34593, 2004.

${ }^{2}$ International Electrotechnical Commission, "IEC 61400-1: Wind Turbines - Part 1: Design Requirements,” Edition 3, 2005.

${ }^{3}$ Kelley, N.D., Jonkman, B.J., Scott, G.N., Bialasiewicz, J.T., and Redmond, L.S., "The Impact of Coherent Turbulence on Wind Turbine Aeroelastic Response and Its Simulation," Wind Power 2005 Conference Proceedings, May 15-18, Denver, CO, American Wind Energy Association, 2005.

${ }^{4}$ Basu, S., Porté-Agel, F., "Large-eddy simulation of stably stratified atmospheric boundary layer turbulence: a scaledependent dynamic modeling approach," Journal of the Atmospheric Sciences, Vol. 63, 2074-2091, 2006.

${ }^{5}$ Jonkman, B. J. and Buhl, M.L., Jr., "TurbSim User's Guide for Version 1.30," National Renewable Energy Laboratory, 2008.

${ }^{6}$ Jonkman, J. M. and Buhl, M. L. Jr., "FAST User's Guide,” National Renewable Energy Laboratory, NREL/EL-500-38230, Golden, CO, 2005.

${ }^{7}$ Smagorinsky, J., "General circulation experiments with the primitive equations," Mon. Wea. Rev., 91, 99-164, 1963.

${ }^{8}$ Geurts, B. J., Elements of Direct and Large-eddy Simulation. Edwards, Philadelphia, 329 pp., 2003.

${ }^{9}$ Lilly, D. K., "A proposed modification of the Germano subgrid-scale closure method," Phys. Fluids A, 4, 633-635, 1992.

${ }^{10}$ Germano, M., U. Piomelli, P. Moin, andW. H. Cabot, "A dynamic subgrid-scale eddy viscosity model,” Phys. Fluids A, 3, $1760-1765,1991$.

${ }^{11}$ Anderson, W. C., S. Basu, and C. W. Letchford, "Comparison of dynamic subgrid-scale models for simulations of neutrally buoyant shear-driven atmospheric boundary layer flows," Environ. Fluid Mech., 7,pp. 195-215, 2007.

${ }^{12}$ Basu, S., J.-F. Vinuesa, and A. Swift, "Dynamic LES modeling of a diurnal cycle,” J. Appl. Meteorol. Climatol., 47, 11561174, 2008.

${ }^{13}$ Jonkman, J. M., Butterfield, S., Musial, W. and Scott, G., "Definition of a 5MW Reference Wind Turbine for Offshore System Development," National Renewable Energy Laboratory, NREL/TP-500-38060, Golden, Colorado, 2007.

${ }^{14}$ Basu, S., Foufoula-Georgiou, E., and Porté-Agel, F., "Synthetic turbulence, fractal interpolation, and large-eddy simulation," Physical Review E, 026310, 2004.

${ }^{15}$ ASTM, "Standard Practices for Cycle Counting in Fatigue Analysis," American Society for Testing and Materials Standards, E1049-85, 1985. 